\title{
Das Konzept raumplanerischer Naturgefahrenprävention im Schweizer Kanton Graubünden
}

\section{Kerstin Sauerbrey, Nürnberg}

\section{Einleitung}

In der Vergangenheit verursachten Naturereignisse in den Alpen immer wieder erhebliche Schäden an Personen und Sachgütern. Diese sowie die zu erwartenden Auswirkungen einer globalen Erwärmung im Alpenraum führen den betroffenen Staaten die zunehmende Notwendigkeit einer effizienten Naturgefahrenabwehr vor Augen. Während man im Bereich der Naturgefahrenprävention anfangs vor allem auf technische Lösungen setzte, ist man in den letzten Jahren vermehrt dazu übergegangen, dem bisherigen Instrumentarium präventiv-raumplanerische Maßnahmen hinzuzufügen. In der Schweiz bemüht man sich nicht zuletzt aufgrund des katastrophalen Lawinenwinters der Jahre 1950/51 bereits seit Mitte der 50er Jahre des 20. Jahrhunderts um die Integration und Umsetzung raumplanerischer Maßnahmen zum Schutz vor Naturgefahren. Der Grundstein zur Ausweisung von Lawinengefahrenzonen wurde im Jahre $1952 \mathrm{mit}$ den «Richtlinien betreffend Aufforstungs- und Verbauungsprojekte in lawinengefährdeten Gegenden» des EIDGENösSISChEN Departments DES INNEREN (EDI) gelegt. Das Eidgenössische Institut für Schneeund Lawinenforschung Weissfluhjoch-Davos (EISLF) begann 1955 mit der Erfassung von Lawinenkatastern. Mit der Ausweisung von Lawinengefahrenzonen wurde erst einige Jahre später begonnen, basierend auf der im Oktober 1965 durch den Art. 32 Abs. 2 ergänzten Vollziehungsverordnung zum Eidgenössischen Forstpolizeigesetz (vgl. BaUMANN \& BURI 1994). Im Jahre 1975 publizierte das Bundesamt Für Forstwesen heute integriert in das Bundesamt für Umwelt, Wald und Landschaft (BUWAL) - provisorische «Richtlinien zur Berücksichtigung der Lawinengefahr beim Erstellen von Bauten und bei Verkehrs- und Siedlungsplanung» (vgl. BUNDESAMT Für FoRSTWESEN (BFF) 1975). In den folgenden Jahren wurde die Beurteilung von Naturgefahren präzisiert und auf alle gravitativen Naturgefahren ausgedehnt.

Das Bundesgesetz über die Raumplanung trat im Jahre 1979 in Kraft und zwingt seitdem Bund, Kantone und Gemeinden, bei ihren raumwirksamen Tätigkeiten natürliche Gegebenheiten und damit alle Naturgefahren zu berücksichtigen (vgl. Art. 1 RPG). Gemäß Art. 6 Abs. 2 RPG sind die Kantone dazu verpflichtet, in denjenigen Gebieten Gefahrenzonen auszuweisen, die durch Naturgefahren erheblich bedroht sind. Diese gesetzlichen Bestimmungen erhielten im Jahre 1991 ein zusätzliches Gewicht durch das Wasserbau- (WBG) und Waldgesetz (WaG). Durch das Inkrafttreten der beiden Gesetze wurde raumplanerischen Präventionsmaßnahmen eine rechtsverbindliche Vorrangstellung vor technischen Schutzmaßnahmen eingeräumt.

Im vorliegenden Beitrag wird - ausgehend von der Analyse raumordnerisch-rechtlicher und raumplanungsrelevanter sachrechtlicher Bestimmungen gezeigt, in welchem Maße Naturgefahren im Raumplanungskonzept der Schweiz Berücksichtigung finden. Es wird dargelegt, welchen Beitrag die raumplanerischen Instrumentarien der verschiedenen Planungsebenen zum Schutz vor Naturgefahren leisten. Da sich aufgrund des föderativen Selbstverwaltungsrechtes der Schweizerischen Eidgenossenschaft in den einzelnen Kantonen unterschiedliche raumplanerische Präventionskonzepte entwickelten, wird in diesem Artikel die passive Präventionspraxis des Kantons Graubünden analysiert und bewertet.

\section{Maßnahmen zum Schutz vor Naturgefahren}

Schäden entstehen durch das Einwirken von Naturereignissen auf das anthropogene Nutzungssystem. Da nutzbare Flächen im Alpenraum eine begrenzte Ressource darstellen, führen wachsender Siedlungs- und Erschließungsdruck zu einer zunehmenden Exposition von Bevölkerung und Sachwerten gegenüber Naturgefahren. Eine Minimierung oder Vermeidung von Schäden kann zum einen durch technische Einflussnahme auf die Gefahrenquelle selbst erreicht werden und zum anderen durch eine der jeweiligen Gefahrensituation angepasste Nutzung gefahrenexponierter Flächen.

Schutzmaßnahmen an der Gefahrenquelle zielen auf eine Verringerung der Eintrittswahrscheinlichkeit eines Naturereignisses ab. Negative Auswirkungen auf den menschlichen Lebens- und Wirtschaftsraum sollen durch die Beeinflussung und Kontrolle von Prozessbildung und Materialtransport reduziert oder gänzlich verhindert werden. Derartige technische Maßnahmen stellen eine kostenintensive Anpassung des Gefahrenpotentials an die menschliche Nutzung dar. Das Schadenspotential selbst bleibt dabei unbeeinflusst. Während beispielsweise durch eine Lawinenverbauung das Risiko für Menschen und Gebäude reduziert wird, 
von einer Lawine heimgesucht zu werden, vergrößert sich das Schadenspotential in der Regel, da im entsprechenden Gefahrengebiet eine Bautätigkeit im Allgemeinen weiterhin stattfindet. Die Siedlungsentwicklung erhält damit keine angemessene präventive Revision (vgl. Egli 1996; Bloetzer et al. 1998).

Schutzmaßnahmen am gefährdeten Objekt beeinflussen das Schadensausma $ß$ des jeweiligen Naturereignisses. Durch eine an die Gefahrensituation angepasste Wahl der Nutzungsart und -intensität sowie der Bauweise können eventuelle Schäden reduziert und somit das Schadenpotential verkleinert werden. Dabei übernimmt die Raumplanung eine entscheidende Rolle, da sie die Raum- bzw. Flächennutzung festlegt, die für das Schadenspotential die entscheidende Größe ist. Die Ausdehnung eines Siedlungsgebietes innerhalb eines potentiellen Gefahrengebiets wird durch eine entsprechende Auszonung von noch nicht überbauten Arealen verhindert. Bei existierenden Bauten müssen im Rahmen von Umoder Erweiterungsvorhaben Objektschutzmaßnahmen ergriffen werden, welche die Schadensempfindlichkeit des Gebäudes herabsetzen. Im Gegensatz zu Maßnahmen an der Gefahrenquelle, deren Wirkung kurzfristig eintritt, ist die Wirkung von raumplanerischen Maßnahmen am gefährdeten Objekt langfristig angelegt, da sie erst im Zusammenhang mit dem nächsten Um- oder Neubau durchgesetzt werden können (vgl. Egli 1996; BloETZER et al. 1998).

«Raumplanung wird als ein System räumlicher Planung verstanden, das die Nutzung des Raumes normativ regelt» (Greiving 2002: 136).

Danach ist es Aufgabe der Raumplanung, auf der Grundlage gesetzlicher Bestimmungen und mit Hilfe des raumplanerischen Instrumentariums, raumrelevante Vorgänge und Entscheide zu koordinieren und die gesetzlich zulässigen Nutzungsarten verbindlich festzusetzen, wobei die unterschiedlichen Nutzungsansprüche an den Raum berücksichtigt werden können. Während der Aufgabenbereich räumlicher Gesamtplanung überfachlich angelegt ist, übernehmen die verschiedenen Fachplanungen die Lösung der ihnen vom Gesetzgeber aufgetragenen spezifisch-fachlichen Obliegenheiten.

Zum Zwecke der Naturgefahrenabwehr ist die Raumplanung angehalten, auf der Basis vorhandener Erkenntnisse über Naturgefahren und mit Hilfe ihrer raumplanerischen Instrumente die notwendigen Maßnahmen zu ergreifen, um Nutzungsansprüche an die vorhandenen Gefahrenpotentiale anzupassen und damit Schäden von vornherein zu vermeiden. Fachplanungen haben in diesem Zusammenhang die Aufgabe, das fachliche Know-how bezüglich der Gefahrensituation zu erarbeiten und als Grundlage für die raumplanerische Umsetzung bereitzustellen.

\section{Naturgefahren und Raumplanung in der Schweiz}

In der Schweiz unterliegen der Bund, die Kantone und die Gemeinden der Planungspflicht (vgl. Art. 2 Abs. 1 RPG). Der Erlass eines raumplanerischen Grundsatzgesetzes (Bundesgesetz über die Raumplanung (RPG)), die Zusammenarbeit mit den Kantonen sowie die Koordination und Förderung kantonaler Bestrebungen liegen gemäß Art. 75 BV im Aufgabenbereich des Bundes. Dieser erfüllt seine Planungs- und Abstimmungspflicht im Rahmen der behördenverbindlichen Planung mit Hilfe von Konzepten und Sachplänen (vgl. Art. 13 RPG).

Entsprechend dem föderativen Selbstverwaltungsrecht der Schweizerischen Eidgenossenschaft liegt die Hauptverantwortung für die Raumplanung bei den Kantonen. Als raumplanerisches Instrument sieht das Bundesgesetz über die Raumplanung den kantonalen Richtplan (vgl. Art. 6-12 RPG) vor. Dieser dient der räumlichen Ordnung und Vorsorge sowie der vertikalen und horizontalen Koordination aller befassten Stellen (vgl. GiLgeN 1999). Richtpläne besitzen Behördenverbindlichkeit (vgl. Art. 9 Abs. 1 RPG). Das heißt, sie geben den planenden Einrichtungen aller politischen Ebenen verbindliche Richtlinien für die Ausübung ihres Planungsermessens vor.

In der Schweiz verfassen die Gemeinden Bauordnungen, Rahmennutzungs- und Sondernutzungspläne.

«Nutzungspläne lokalisieren, differenzieren und dimensionieren die gesetzlich zulässige Nutzung des Bodens und des damit verbundenen Raumes nach Art und Intensität. Sie treffen die grundlegende Abtrennung der Bauzone von den Nichtbauzonen» (GILGEN 2001: 76).

Gemäß Art. 14 Abs. 2 RPG findet innerhalb der kommunalen Nutzungspläne eine Unterscheidung von Bau-, Landwirtschafts- und Schutzzonen statt. Zudem können im Rahmen der kantonalen Gesetzgebung so genannte «weitere Nutzungszonen» (vgl. Art.18 RPG) als Gefahrenzonen ausgewiesen werden. Nutzungspläne sind nach Art. 21 Abs. 1 RPG für jedermann verbindlich. Damit hat jeder, der Boden nutzen will, sie als rechtlichen Rahmen mit festgelegten Anordnungen zu beachten. Die Baubewilligung, deren Erteilung in den meisten Kantonen ebenfalls im Zuständigkeitsbereich der Gemeinde liegt, stellt ein entscheidendes Instrument zur raumwirksamen Umsetzung kommunaler Nutzungspläne dar (vgl. Art. 22 RPG).

Das Bundesgesetz über die Raumplanung gebietet sowohl dem Bund, den Kantonen als auch den Gemeinden bei ihren raumwirksamen Tätigkeiten die natürlichen Gegebenheiten und damit das Potential von Naturgefahren zu berücksichtigen (vgl. Art. 1 Abs. 1 RPG). Eine zusätzliche Gewichtung und deutli- 


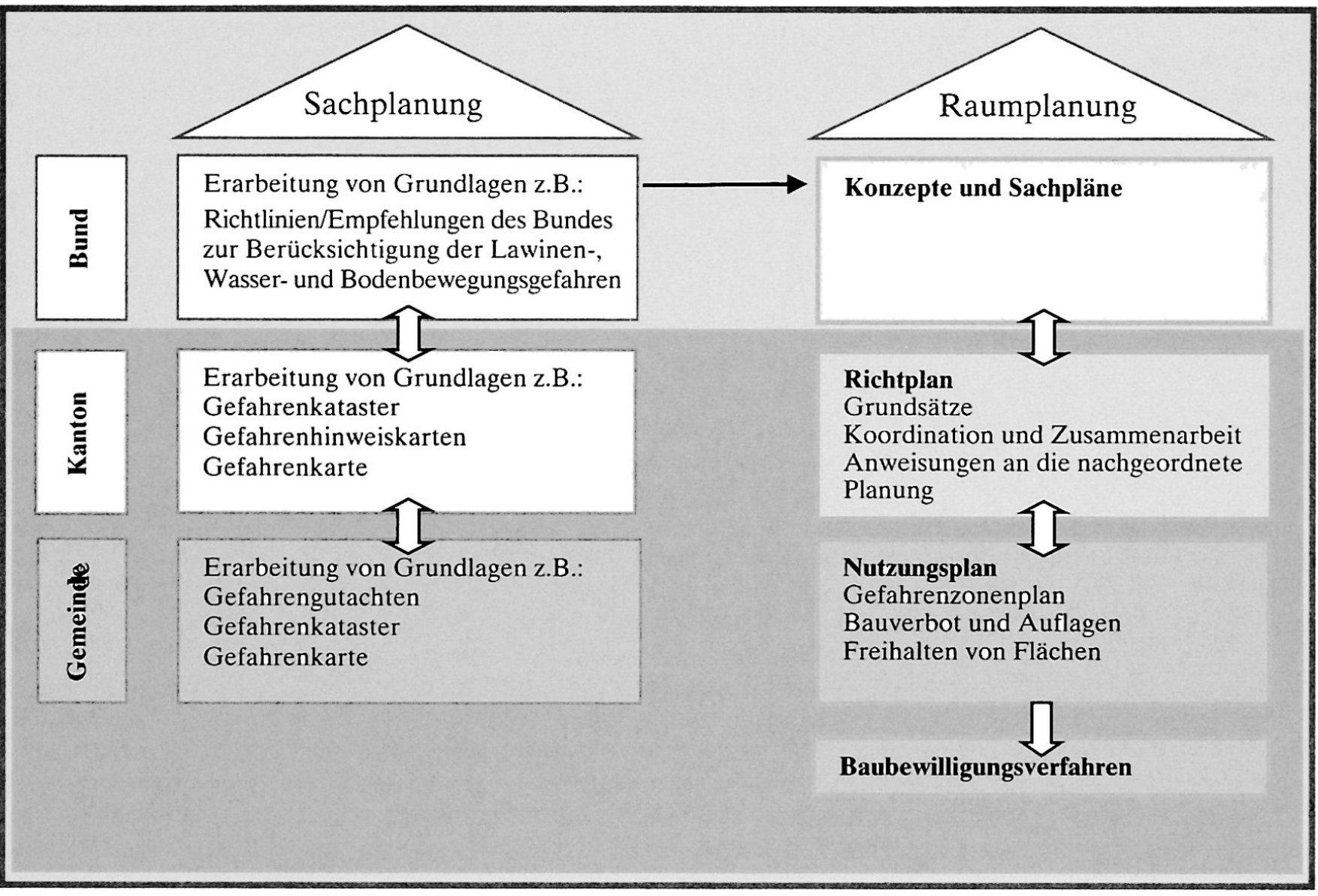

Abb. 1: Die Berücksichtigung von Naturgefahren im Planungssystem der Schweiz Consideration of natural hazards in planning procedures in Switzerland La prise en compte des dangers naturels dans le système d'aménagement de la Suisse Quelle: GugGisberg \& WeGELIN 1998: 4 (verändert)

che Konkretisierung erhalten die raumplanungsrechtlichen Bestimmungen hinsichtlich der Naturgefahrenabwehr durch das Wasserbau- (WBG) und Waldgesetz $(\mathrm{WaG})$ sowie deren entsprechende Verordnungen und Ausführungsbestimmungen.

Im Rahmen raumplanerischer Naturgefahrenprävention stellen in der Schweiz die Richtplanung auf kantonaler Stufe und die Nutzungsplanung auf kommunaler Stufe mit jeweils finanzieller Unterstützung des Bundes die entscheidenden Planungsebenen dar (vgl. Abb. 1).

Im Rahmen der Richtplanung sind die Kantone nach Art. 6 Abs. 2 lit. C RPG verpflichtet, vorhandene Naturgefahren aufzuzeigen und dabei auf mögliche Gefahrenpotentiale hinzuweisen.

Die Ausweisung von Gefahrengebieten kann innerhalb des Richtplans selbst vorgenommen werden. Es besteht allerdings auch die Möglichkeit, auf die
Grundlagenkarten zum Richtplan oder auf die kommunalen Gefahrenzonenpläne zu verweisen (vgl. GuGGISBERG 1994; BUNDESAMT Für UMWELt, WALD UND LANDSCHAFT (BUWAL), BUNDESAMT FÜR WASSERWIRTSCHAFT (BWW) \& BUNDESAMT FÜR RAUMPLANUNG (BRP) 1997a).

Die Behördenverbindlichkeit des kantonalen Richtplans verlangt, Naturgefahren auch in der kommunalen Nutzungsplanung zu berücksichtigen. Der Nutzungsplan mit seiner eigentümerverbindlichen und parzellengenauen Festlegung von Ort, Zweck und Umfang der Nutzung ist das zentrale Instrument zur Umsetzung raumplanerischer Naturgefahrenprävention und somit zur Begrenzung des Schadenspotentials.

Während in Gebieten mit starker Gefährdung durch Naturgefahren grundsätzlich keine Neubauten zugelassen werden, sind bei weniger starker Gefährdung bauliche Tätigkeiten mit entsprechenden Auflagen möglich. 
Die Berücksichtigung von Gefahrenkarten in der kommunalen Nutzungsplanung ergibt sich aus den Vorgaben des kantonalen und kommunalen Rechts. So existieren von Kanton zu Kanton unterschiedliche Lösungen hinsichtlich der Berücksichtigung von Naturgefahren in der kommunalen Nutzungsplanung. In einigen Kantonen werden Gefahrengebiete durch eine abgestimmte Zonierung im Nutzungsplan berücksichtigt. Andere Varianten sind die Ausweisung von überlagerten Gefahrenzonen oder die rechtskräftige Genehmigung eines speziellen separaten Gefahrenzonenplans (vgl. KIENHOLZ 1999; BLOETZER et al. 1998).

\section{Die raumplanerische Präventionspraxis des Kantons Graubünden}

Die wesentliche Voraussetzung für eine effektive Umsetzung raumplanerischer Präventionsmaßnahmen ist die Erkennung und Dokumentation der Naturgefahrensituation sowie deren Beurteilung und Bewertung (vgl. HüBL et al. 2002).

Im Kanton Graubünden konzentriert sich diese Grundlagenerarbeitung auf so genannte Erfassungsbereiche. In diesen nach einheitlichen Kriterien definierten Bereichen werden Grundlagendaten zur Gefährdung erhoben, aufbereitet und analysiert, um schließlich in die Ausweisung von Gefahrenzonen zu münden (vgl. Amt für Wald Graubünden (AfW) 1999). Für jede Gemeinde des Kantons werden Erfassungsbereiche mit vollständigem oder unvollständigem Ereigniskataster ausgewiesen, wobei deren Festlegung auf einem Entscheidungsraster mit Objektkategorien und Abstandsrichtwerten basiert (vgl. CAPREZ 2000 und Tab. 1). Während beim Erfassungsbereich mit vollständigem Ereigniskataster alle innerhalb eines definierten Areals eingetretenen Naturereignisse aufgezeichnet werden, finden beim Erfassungsbereich mit unvollständigem Ereigniskataster ausschließlich Naturereignisse mit Schadenfolge Berücksichtigung (vgl. Amt Für Wald Graubünden (AfW) 1999).

Im Jahre 1999 begann das Amt für Wald Graubünden mit der kommunalen Abgrenzung der Erfassungsbereiche für den gesamten Kanton. Von Anfang an wurde eine enge Zusammenarbeit mit dem Amt für Raumplanung im Rahmen des Festsetzungsverfahrens angestrebt, um auch raumplanerische Anliegen bei der Festlegung berücksichtigen zu können (vgl. WILHELM 2001).

Die in den ausgewiesenen Erfassungsbereichen geführten Ereigniskataster sind Verzeichnisse eingetretener Naturereignisse. Sie umfassen die Abläufe und die festgestellten Schäden, die Wirkungsbereiche sowie die meteorologischen und hydrologischen Daten (vgl.
Amt für Raumplanung Graubünden (ARP) \& Amt Für Wald Graubünden (AfW) 2001). Seit Anfang 2002 führt der Kanton Graubünden die Ereignisdokumentation EDV-gestützt durch (StorMe) (vgl. BurreN \& TAVERna 2001). Die im Ereigniskataster festgehaltenen Angaben liefern die Basis für die Definition potentieller Gefahrenbereiche.

Die Resultate der Gefahrenbeurteilung bzw. -bewertung durch die Gefahrenkommission werden zunächst in den Gefahrenhinweiskarten und den Gefahrenkarten festgehalten.

Soll die Naturgefahrensituation im Rahmen der Raumnutzung berücksichtigt werden, ist eine abgestufte Gefahrenbeurteilung notwendig, da die kantonale und die kommunale Planungsebene jeweils unterschiedliche Anforderungen an die fachliche Tiefe und den Detaillierungsgrad stellen (vgl. Abb. 2). Während die kantonale Richtplanung regionale Übersichten bzw. generelle Aussagen über die Gefahrensituation benötigt, verlangt die kommunale Nutzungsplanung exakte Detailangaben.

Die Gefahrenhinweiskarten bilden «eine Grundlage für die großräumige Groberkennung der Gefährdungssituation» (AMT FÜR RAUMPLANUNG GRAUBÜNDEN (ARP) \& AmT FÜR Wald GRAubüNdEN (AfW) 2001: 3). Sie enthalten zwar Informationen über Gefahrenarten, geben allerdings keine Auskunft über den Gefährdungsgrad (Eintrittswahrscheinlichkeit und Intensität). Die Gefahrenhinweiskarte besitzt nur eine geringe Bearbeitungstiefe. Sie dient vorwiegend als Planungsgrundlage für die Festlegung der Notwendigkeit und Dringlichkeit spezifischer Detailuntersuchungen und genügt damit den Ansprüchen der Richtplanung.

Die Gefahrenkarte hingegen enthält genaue Aussagen über die Gefahrenart, die räumliche Ausdehnung und den Grad der Gefährdung. Prozessgetrennt wird sie vom Amt für Wald für die Gefahrenarten Lawine, Wasser, Sturzprozesse und Rutschungen geführt. Als Grad der Gefährdung werden die Intensität und die Wahrscheinlichkeit (Häufigkeit und Wiederkehrdauer) der jeweiligen Gefahrenart festgelegt. Dabei wird zwischen drei Gefahrenstufen unterschieden, wobei die Schadenwirkung für jede Gefahrenart und Gefahrenstufe aufgezeigt wird. Die Abstufung erfolgt von einem Hinweisbereich (geringe Gefährdung) über einen Gebotsbereich (mittlere Gefährdung) bis hin zu einem Verbotsbereich (erhebliche Gefährdung). Aufgrund ihrer hohen Bearbeitungstiefe (parzellengenaue Abgrenzung) stellt die Gefahrenkarte die entscheidende Planungsgrundlage für die Ausweisung von Gefahrenzonen im Rahmen der Nutzungsplanung dar (vgl. Amt für Raumplanung Graubünden (ARP) \& 
Amt für Wald Graubünden (AfW) 2001). (Gefahrenkarten des Amtes für Wald Graubünden sind unter folgenden Internet-Adressen zu finden: Muster-Gefahrenkarte Lawinen: http://www.wald.gr.ch/download/ gko_7_3.pdf; Muster-Gefahrenkarte Sturzprozesse: http://www.wald.gr.ch/download/1-1-1-4_GKo_10_3.pdf; Muster-Gefahrenkarte Wasser: http://www.wald.gr.ch/ download/GKo_8_4.pdf.)

Erst durch die Einbindung der Gefahrenkarten in den kommunalen Nutzungsplan kommt es zu einer rechtsverbindlichen Berücksichtigung von Naturgefahren in der Raumplanung (Ausweisung von Gefahrenzonen) und damit zu einer der Gefahrensituation angepassten Nutzungsfestlegung. Die grundeigentümerverbindliche Festlegung der Gefahrenzonen in Form überlagerter Nutzungszonen findet nach Art. 37 des Raumplanungsgesetzes für den Kanton Graubünden (KRPG) in den Nutzungsplänen der Gemeinden statt. Art. 16 KRPG unterscheidet zwei Gefahrenzonen:

- Gefahrenzonen hoher Gefahr (rote Gefahrenzone) "In der Zone mit hoher Gefahr dürfen keine Bauten erstellt und erweitert werden, die dem Aufenthalt von Menschen und Tieren dienen. Zerstörte Bauten dürfen nur in Ausnahmefällen wieder aufgebaut werden. Standortgebundene Bauten, die nicht dem Aufenthalt von Menschen und Tieren dienen, sind mit entsprechendem Objektschutz zulässig.» (Art. 17 Abs. 1 KRVO)

- Gefahrenzonen geringerer Gefahr (blaue Gefahrenzone)

«In Zonen mit geringer Gefahr bedürfen Bauvorhaben (Neu- und Erweiterungsbauten, Umbauten mit erheblicher Wertvermehrung) der Genehmigung durch die Gebäudeversicherung des Kantons Graubünden. Bei Standortgefährdung umschreibt diese die erforderlichen baulichen Schutzmaßnahmen als Bauauflagen.» (Art. 17 Abs. 2 KRVO)

Die Begutachtung und Ausweisung von Gefahrenzonen führen in Graubünden die jeweils zuständigen Gefahrenkommissionen durch. Die grundeigentümerverbindliche Festlegung von Gefahrenzonen sowie die Verabschiedung des bereinigten Gefahrenzonenplanes liegen im Zuständigkeitsbereich der Gemeinden. Erlass und Änderungen der im Zonenplan integrierten Gefahrenzonen müssen durch die Regierung genehmigt werden. Die Überprüfung, Anpassung und Nachführung der Gefahrenzonenpläne wird bei der Revision von Zonenplänen, bei der Erhöhung bzw. beim Neueintritt der Gefährdung durch Naturereignisse oder aufgrund neuer wissenschaftlicher Erkenntnisse sowie bei der Verminderung der Gefährdung infolge von Schutzmaßnahmen durchgeführt (vgl. Art. 13 ff. der Richtlinien für die Gefahrenzonenplanung). Wird eine neue oder eine erhöhte Gefährdung durch Naturereignisse festgestellt, muss das neu zu beurteilende Areal abgegrenzt und durch die Gefahrenkom- mission überprüft werden. Es erfolgt der Erlass einer Bausperre durch die Gemeinde nach Art. 56 KRPG sowie die Information der Gebäudeversicherung und des Amtes für Raumplanung.

\section{Die Rolle der Gebäudeversicherung}

Innerhalb raumplanerischer Naturgefahrenprävention nimmt die Gebäudeversicherung des Kantons Graubünden (GVA) einen besonderen Stellenwert ein. Durch ihre Beteiligung am Raumplanungsverfahren zur Ausweisung und Freihaltung bzw. risikogerechten Bebauung von Gefahrenzonen wird die GVA zur Mitbeteiligten bei der Umsetzung des kantonalen Raumplanungsgesetzes. Die Gebäudeversicherung unterzieht Bauvorhaben in Gefahrenzonengebieten einer besonderen Prüfung. Da die Gebäudeversicherung im Kanton Graubünden eine Monopolstellung besitzt und für jedermann eine Versicherungspflicht für Elementarrisiken besteht (vgl. Art. 4 Abs. 1 GVAG), müssen die von ihr geforderten Auflagen zwingend berücksichtigt werden. Sollten festgelegte Objektschutzmaßnahmen bei Bautätigkeiten ignoriert werden, erlischt der Versicherungsschutz (vgl. Art. 6 GVAG), was eine Aufhebung der Baubewilligung zur Folge haben kann. Es handelt sich bei diesem Vorgehen um eine Aufgabendelegierung des Staates, die im Sinne des Solidaritätsprinzips erfolgt, da auf diese Weise die Gesamtheit aller Versicherten keine unverantwortbaren Einzelrisiken zu übernehmen hat (vgl. BloETZER et al. 1998; FISCHER 2000).

\section{Die Realität raumplanerischer Naturgefahren- prävention in der Schweiz}

Die raumplanerische Naturgefahrenprävention zeigt in den einzelnen Kantonen der Schweiz trotz einheitlicher gesetzlicher Rahmenvorgaben des Bundes deutliche Unterschiede. Neben kantonal heterogenen Naturgefahrensituationen sind diese Varianten im Wesentlichen auf einen unterschiedlichen Konkretisierungs- und Detaillierungsgrad der raumplanungsund sachrechtlichen Bestimmungen in der kantonalen und kommunalen Gesetzgebung, auf verschiedenartige Vorgehensweisen und Resultate im Bereich der Gefahren- und Risikoanalyse sowie auf Unterschiede in der Handhabung des raumplanerischen Instrumentariums zurückzuführen.

Im Kanton Graubünden wurde auf der Grundlage entsprechend konkretisierter kantonaler und kommunaler Gesetzesbestimmungen ein umfassendes Konzept der Gefahrenzonenplanung entwickelt, das eine effektive Umsetzung raumplanerischer Präventionsmaßnahmen erlaubt. 


\begin{tabular}{|c|c|c|c|}
\hline Erfassungsbereich & Objekte & $\begin{array}{l}\text { Anzahl der Gebäude } \\
\text { gleicher oder höherer } \\
\text { Kategorie/Minimale } \\
\text { Abstände }\end{array}$ & $\begin{array}{l}\text { Erreichbarkeit über das } \\
\text { öffentliche Wegnetz } \\
\text { (Kantons- und } \\
\text { Gemeindestraßen) }\end{array}$ \\
\hline $\begin{array}{l}\text { Erfassungsbereich mit } \\
\text { vollständigem } \\
\text { Ereigniskataster }\end{array}$ & $\begin{array}{l}\text { - alle Bauzonen und } \\
\text { ganzjährig } \\
\text { bewohnte/genutzte } \\
\text { Einzelgebäude } \\
\text { - Campinganlagen } \\
\text { - Schießanlagen }\end{array}$ & keine Einschränkungen & $\begin{array}{l}\text { ganzjährig über das } \\
\text { öffentliche Wegnetz } \\
\text { erreichbar }\end{array}$ \\
\hline \multirow[t]{3}{*}{$\begin{array}{l}\text { Erfassungsbereich mit } \\
\text { unvollständigem } \\
\text { Ereigniskataster }\end{array}$} & $\begin{array}{l}\text { - ganzjährig } \\
\text { bewohnte/genutzte } \\
\text { Einzelgebäude }\end{array}$ & \multirow[t]{2}{*}{ keine Einschränkungen } & $\begin{array}{l}\text { nur zeitweise oder nicht } \\
\text { über das öffentliche } \\
\text { Wegnetz erreichbar }\end{array}$ \\
\hline & $\begin{array}{l}\text { - nicht ganzjährig } \\
\text { genutzte Gebäude, dem } \\
\text { Aufenthalt von Mensch } \\
\text { und Tier dienend }\end{array}$ & & $\begin{array}{l}\text { nur zeitweise oder nicht } \\
\text { über das öffentliche } \\
\text { Wegnetz erreichbar }\end{array}$ \\
\hline & $\begin{array}{l}\text { - nicht ganzjährig } \\
\text { genutzte Gebäude, dem } \\
\text { Aufenthalt von Mensch } \\
\text { und Tier dienend }\end{array}$ & $\begin{array}{l}\text { mindestens } 2 \text { Gebäude } \\
\text { im Abstand kleiner als } \\
250 \mathrm{~m} \text { zueinander }\end{array}$ & $\begin{array}{l}\text { nur zeitweise oder nicht } \\
\text { über das öffentliche } \\
\text { Wegnetz erreichbar }\end{array}$ \\
\hline $\begin{array}{l}\text { Erfassungsbereich unter } \\
\text { speziellen } \\
\text { Voraussetzungen }\end{array}$ & $\begin{array}{l}\text { - nicht dem Aufenthalt } \\
\text { von Mensch und Tier } \\
\text { dienende Gebäude } \\
\text { (Heuschober, Remisen } \\
\text { etc.) }\end{array}$ & $\begin{array}{l}\text { Einbezug, falls inner- } \\
\text { halb von } 150 \mathrm{~m} \text { von } \\
\text { einem bereits festge- } \\
\text { legten Erfassungs- } \\
\text { bereich entfernt }\end{array}$ & \\
\hline
\end{tabular}

Tab. 1: Entscheidungsraster des Erfassungsbereiches Naturgefahren

Raster supporting decision-making processes in the area of natural hazards

Grilles de décision relatives à l'inventaire des dangers naturels

Quelle: CAPREZ 2000: 48

Im Rahmen der Naturgefahrenabwehr versteht man es im Kanton Graubünden, den Beitrag der raumplanerischen Instrumente in angemessener Weise zu nutzen. Innerhalb des «Kantonalen Richtplanes Graubünden 2000» werden sowohl die Zukunftsaussichten im Umgang mit Naturgefahren, wesentliche Leitüberlegungen (Zielsetzung, Strategische Schwerpunkte), Grundsätze, zu ergreifende Maßnahmen als auch Verantwortungsbereiche behördenverbindlich festgesetzt. Durch die Festlegung der Verantwortungsbereiche und der zuständigen Fachstellen können im Rahmen einer integrierten Gefahrenabwehr Abstimmungs- und Kompetenzprobleme nahezu ausgeschaltet werden. Die Umsetzung raumplanerischer Präventionsmaßnahmen geschieht im Rahmen der Nutzungsplanung. Hier werden Art und Intensität von Eigentumsbeschränkungen, die oftmals mit finanziellen Konsequenzen verbunden sind, zum Schutz des Nutzungswilligen parzellengenau und grundeigentümerverbindlich festgelegt. Die Kriterien für die Zuweisung einer Nutzungsparzelle zu einem Gefahrengebiet müssen transparent und nachvollziehbar sein, wenn Gefahrenzonen Akzeptanz finden und respektiert werden sollen.

Deshalb werden Grundlagen erarbeitet und bereitgestellt, die in räumlicher und zeitlicher Hinsicht sowie in ihrer sachlichen Tiefe den Anforderungen der kantonalen Richtplanung und der kommunalen Nutzungsplanung entsprechen. Im Kanton Graubünden ist die Gefahrendokumentation entscheidender Bestandteil der Grundlagenerarbeitung. Die Aufnahme der Erfas- 


\section{Gefahrenhinweiskarte}

Inhalt:

Grobe Übersicht über die Gefährdungssituation

Angabe der Gefahrenart

Großräumige Ausweisung

Bearbeitungstiefe:

Geringe Bearbeitungstiefe

\section{Erfasstes Areal:}

Kanton

\section{Überprüfung:}

Periodisch im Rahmen der Richt- und

Nutzungsplanrevisionen

\section{Nachführung:}

Bei erheblich veränderter Gefahrensituation - infolge von Schutzmaßnahmen

- infolge von Veränderungen der natürlichen Voraussetzungen

\section{Gefahrenkarte}

\section{Inhalt:}

Genaue Angaben zur Gefahrenart,

räumlichen Ausdehnung und zum Grad der

Gefährdung in drei Gefahrenstufen

Detaillierte Dokumentation

Bearbeitungstiefe:

Hohe Bearbeitungstiefe (parzellengenaue

Abgrenzung)

Erfasste Areale:

Konzentration auf besiedelte, erschlossene oder zukünftig zu erschließende Gebiete sowie auf Verkehrswege (Erfassungsbereiche)

\section{Überprüfung:}

Periodisch im Rahmen der Richt- und

Nutzungsplanrevisionen

\section{Nachführung:}

Bei erheblich veränderter Gefahrensituation - infolge von Schutzmaßnahmen

- infolge von Veränderungen der natürlichen Voraussetzungen

\section{Kantonaler Richtplan}

\section{Inhalt bezüglich Gefahren:}

Abstimmungsanweisungen zum weiteren

Vorgehen im Text; eventuell grobe

Abgrenzung der Gefahrengebiete in der

Karte

Grobe Berücksichtigung der Gefahrengebiete

\section{Nachführung:}

Ergänzung bzw. Nachführung bei veränderten Verhältnissen oder neuen Aufgaben; gesamthafte Überprüfung der Richtplanung und nötigenfalls Überarbeitung alle $10 \mathrm{Jahre}$

\section{Kommunaler Nutzungsplan}

Inhalt bezüglich Gefahren:

Berücksichtigung der in der Gefahrenkarte bezeichneten Gefahrengebiete nach Gefahrenarten, Gefahrenstufen und entsprechenden Konsequenzen für die Nutzung

Parzellengenaue Berücksichtigung der Gefahrengebiete

\section{Nachführung:}

Nachführung bei Erhöhung der Gefährdung durch Naturereignisse oder bei nachweisbarer Verringerung aufgrund erstellter Schutzmaßnahmen; bei

Totalrevision des Zonenplans (ca. alle 10 15 Jahre)

Abb. 2: Stufengerechte Gefahrenbeurteilung

Multi-step hazard evaluation

Evaluation des dangers en fonction de leurs niveaux

Quelle: Bundesamt für Wasserwirtschaft (BWW), BundeSamt für Raumplanung (BRP) \& Bundesamt Für UMWELT, WALD UND LANDSCHAFT (BUWAL) 1997b: 15/23 (verändert) 
sungsbereiche sowie die Führung des Ereigniskatasters Naturgefahren sind bereits flächendeckend für den gesamten Kanton organisiert. Erste Erfahrungen aus der Praxis zeigen, dass die Daten fortlaufend aktualisiert, angepasst und unterhalten werden müssen. Im Bereich der Gefahrenanalyse ist mit der prozessgetrennten Gefahrenkarte eine geeignete fachliche Grundlage für die raumplanerische Umsetzung gewählt worden. Die Ausarbeitung der Gefahrenkarte, die bisher noch nicht für alle gravitativen Naturgefahren prozessgetrennt vorliegt, soll in Zukunft intensiviert werden. Dabei ist es von Vorteil, dass durch die evaluierende Gefahrenkommission, die sowohl mit Mitarbeitern vor Ort als auch durch solche der Fachstelle Naturgefahren im Amt für Wald besetzt ist, eine überregional standardisierte und der jeweiligen Situation vor Ort angepasste Gefahrenerhebung und -bewertung entsteht.

Der kommunale Nutzungsplan weist Gefahrenzonen als so genannte überlagerte Nutzungszonen aus. Im kommunalen Baugesetz sind der Zweck der Gefahrenzonen definiert und Eigentumsbeschränkungen festgeschrieben. Schutzvorkehrungen werden meist durch eine entsprechende Verfügung im Baubewilligungsverfahren angeordnet. Der Vorteil dieser Vorgehensweise besteht darin, dass Naturgefahren über das gesamte Gemeindegebiet nach einheitlichen Kriterien in der Nutzungsplanung Berücksichtigung finden. Zudem wird mit Hilfe entsprechender kantonaler Anordnungen gewährleistet, dass nicht begründbare Unterscheidungen zwischen einzelnen Gemeinden vermieden werden. Als Nachteil erscheint, dass bei Änderungen des Gefahrenpotentials immer eine Nutzungsplananpassung notwendig wird. Wenn man davon ausgeht, dass in Zukunft die Dynamik von Raumnutzung und Gefährdung durch Naturereignisse zunehmen wird, dann wird es notwendig werden, kurzfristig flexible Anpassungen (Gefahrenzonenausweisung, Nutzungseinschränkungen) vorzunehmen. Mit der gegenwärtigen grundeigentümerverbindlichen Festlegung von Gefahrenzonen in der Nutzungsplanung und den damit verbundenen Abstimmungs- und Genehmigungsprozessen ist eine flexible und kurzfristige Reaktion nicht möglich. Auch ein angeordneter Planungsstopp ist längerfristig nur mit großem juristischem Aufwand zu bewirken. Insoweit wird die prozessorientierte Gefahrenzonenplanung Graubündens einer dynamischen Risikoentwicklung nur beschränkt gerecht.

Um den Ablauf von bisher eher trägen Abstimmungs- und Genehmigungsvorgängen in Zukunft zu beschleunigen, ist es unumgänglich, neben der horizontalen insbesondere die vertikale Integration aller befassten Institutionen zu verbessern. Dazu gehört beispielsweise die Verschlankung von Kooperationsund Koordinationsstrukturen.
Zudem sind rechtliche Ergänzungen und Präzisierungen innerhalb des Raumplanungsgesetzes vorzunehmen, um den beteiligten Instanzen Kompetenzen und Mittel zu übertragen, damit diese Ausweisungen von Gefahrenzonen im Sinne des allgemeinen Interesses schneller durchsetzen können.

Die Einbindung der Gebäudeversicherungsanstalt Graubündens in die raumplanerische Naturgefahrenabwehr zeigt sich als sehr positiv, da die Versicherungsanstalt auf der Basis ihrer Monopolstellung und der obligatorischen Versicherungspflicht für Elementarrisiken ganz wesentlich zur Umsetzung raumplanerischer Naturgefahrenabwehr beiträgt.

Vorteilhaft ist zudem, dass die Naturgefahrenabwehr im Kanton Graubünden eine Querschnittsaufgabe ist. Aufgrund der Beteiligung verschiedener Fachinstitutionen kann die raumplanerische Umsetzung auf spezialisiertes Fachwissen zurückgreifen. Der durch diese Zusammenarbeit hervorgerufene Wissens- und Informationstransfer zwischen allen Beteiligten schlägt sich unmittelbar in der Qualität der Risikovorsorge nieder.

Leider ist die Ausweisung von Gefahrenzonen entsprechend dem aktuellen Konzept nicht flächendeckend für den gesamten Kanton Graubünden vorhanden. In einigen Kommunen bestehen deutliche Rückstände bei der Berücksichtigung der Naturgefahren Wasser und Murgang. Ursache hierfür ist, dass bei der Ausweisung von Gefahrenzonen lange Zeit ausschließlich die Lawinengefahr bedacht wurde. Die Überarbeitung der Gefahrenkarten sowie die entsprechende Übernahme in die kommunalen Nutzungspläne sind allerdings im Gange.

Wie schon in der Vergangenheit wird es auch in Zukunft notwendig sein, das bestehende raumplanerische Präventionskonzept im Hinblick auf sich verändernde Rahmenbedingungen zu überprüfen und gegebenenfalls schnell und flexibel anzupassen.

\section{Literatur}

Amt für Raumplanung Graubünden (ARP) \& Amt Für Wald Graubünden (AfW) (2001): Gefahrenzonen. Planungsrechtliche Umsetzung unter Berücksichtigung der forstlichen Interessen. - Chur.

Amt Für Wald GraubüNden (AfW) (1999): Instruktion und Leitfaden zur Festlegung von Erfassungsbereichen. - Chur (internes Papier).

Baumann, R. \& H. Buri (1994): Erfahrungen mit den Richtlinien zur Berücksichtigung der Lawinengefahr. - In: Informationsheft Raumplanung 1:29-30.

Bloetzer, W., Egli, T., Petrascheck, A. \& J. Sauter (1998): Klimaänderungen und Naturgefahren in der Raumplanung. - Zürich: vdf Hochschulverlag AG an der ETH Zürich. 
Bundesamt Für Forstwesen (BFF) (1975): Richtlinien zur Berücksichtigung der Lawinengefahr beim Erstellen von Bauten und bei Verkehrs- und Siedlungsplanung. - Bern.

BUNDESAMT FÜR UMWELT, WALD UND LANDSCHAFT (BUWAL), BUNDESAMT FÜR WASSERWIRTSCHAFT (BWW) \& BUNDESAMT FÜR RAUMPLANUNG (BRP) (1997a): Empfehlungen zur Berücksichtigung der Massenbewegungen bei raumwirksamen Tätigkeiten. - Bern.

BUNDESAMT Für WASSERWIRTSCHAFT (BWW), BUnDESAMT FÜr RaUmPlanUNG (BRP) \& BUndesamt FÜr UMWELT, WALD UND LANDSCHAFT (BUWAL) (1997b): Empfehlungen zur Berücksichtigung der Hochwassergefahr bei raumwirksamen Tätigkeiten. - Bern.

Burren, S. \& E. Taverna (2001): StorMe. Der informatikgestützte Ereigniskataster im Kanton Graubünden. - In: Bündnerwald 5: 64-72.

CAPREZ, G. (2000): Erfassungsbereiche. - In: Bündnerwald 5: 47-49.

Eidgenössisches Departement des InNeren (EDI) (1952): Richtlinien betreffend Aufforstungs- und Verbauungsprojekte in lawinengefährdeten Gegenden. Bern.

EGLI, T. (1996): Naturgefahren in der Raumplanung. - In: Vermessung, Photogrammetrie, Kulturtechnik 8: 427-432.

Fischer, M. (2000): Gebäudeversicherung und die Naturgefahren. - In: Bündnerwald 5: 21-23.

Gilgen, K. (1999): Kommunale Raumplanung in der Schweiz. - Zürich: vdf Hochschulverlag AG an der ETH Zürich.

Gilgen, K. (2001): Kommunale Richt- und Nutzungsplanung. - Zürich: vdf Hochschulverlag AG an der ETH Zürich.

Greiving, S. (2002): Räumliche Planung und Risiko. München: Gerling Akademie Verlag.

GugGisBERG, C. (1994): Schutz vor Naturgefahren mit Instrumenten der Raumplanung. - In: Informationsheft Raumplanung 1:6-7.

GuggisberG, C. \& F. Wegelin (1998): Welchen Beitrag können die Instrumente der Raumplanung leisten? - Tagungspublikation der Fachtagung Warth (TG): Naturgefahren - Umsetzung in der Raumplanung. Bern: 1-3.

Hübl, J., Kienholz, H. \& A. Loipesberger (eds) (2002): DOMODIS - Documentation of Mountain Disasters, State of Discussion in the European Mountain Areas. - In: Internationale Forschungsgesellschaft Interpraevent 1. - Klagenfurt: International Research Society Interpraevent: 1-36.

KienHOlz, H. (1999): Anmerkungen zur Beurteilung von Naturgefahren in den Alpen. - In: Relief, Boden, Paläoklima 14: 165-184.

Wilhelm, C. (2001): Ziele, geplante Aktivitäten und Fragen zum Umgang mit Naturgefahren im Kanton Graubünden. - In: Bündnerwald 5: 53-63.

\section{Gesetzliche Grundlagen}

Bundesverfassung der Schweizerischen Eidgenossenschaft (BV) vom 18.04.1999 (Stand am 14.05.2002).

Bundesgesetz über die Raumplanung (RPG) vom 22.06.1979 (Stand am 22.08.2000).

Bundesgesetz über den Wald (WaG) vom 04.10.1991 (Stand am 21.12.1999).

Bundesgesetz über den Wasserbau (WBG) vom 21.06.1991 (Stand am 01.01.1995).

Gesetz über die Gebäudeversicherung im Kanton Graubünden (GVAG) vom 12.04.1970 (Stand am 26.11.2002).

Raumplanungsgesetz für den Kanton Graubünden (KRPG) vom 20.05.1973 (Stand am 01.01.1998).

Raumplanungsverordnung für den Kanton Graubünden (KRVO) vom 26.11.1986 (Stand am 01.01.1998).

Richtlinien für die Gefahrenzonenplanung gemäß Regierungsbeschluss Nr. 969 vom 06.05.1997 (Kanton Graubünden).

Vollziehungsverordnung zum Eidgenössischen Forstpolizeigesetz (1965).

\section{Planungsgrundlagen}

Kantonaler Richtplan Graubünden 2000 (genehmigt am 19.09.2003).

\section{Zusammenfassung: Das Konzept raumplanerischer Naturgefahrenprävention im Schweizer Kanton Graubünden}

Die Raumplanung soll im Rahmen der Daseinsfürsorge fortlaufend ihren Beitrag zum Schutz vor Naturgefahren für Menschen und ihre Sachgüter leisten. Ziel einer effektiven passiven Naturgefahrenprävention muss es sein, das Gefährdungsrisiko für Menschen und ihre materiellen Werte unter Wahrung der ökonomischen, sozialen und ökologischen Verhältnismäßigkeit mit gezielten raumplanerischen Maßnahmen auf ein Restrisiko zu reduzieren, um einen gesicherten Wirtschaftsund Lebensraum zu gewährleisten. Am Beispiel der Schweiz und ihres Kantons Graubünden wird im Rahmen dieses Beitrages gezeigt, dass die Raumplanung durchaus in der Lage ist, einen entsprechenden Beitrag im Rahmen der raumplanerischen Vorsorge zu leisten.

\section{Summary: Regional Policy Concept of Natural Hazard Prevention in the Swiss Canton of the Grisons}

Regional planning, within the framework of its provisions, is to consistently contribute towards the protection of people and their property from natural dangers. The objective of an effective passive prevention of natural hazards should be to reduce the hazard risk for people and their property to a minimum by means of clearly targeted regional planning procedures, taking into consideration economic, social and ecological aspects. It should thereby be possible to 
ensure a secure economic and living space. An investigation of Switzerland and its Canton of the Grisons brings to light that regional planning may indeed have a role to play in the prevention of natural hazards.

\section{Résumé: Le concept de prévention des dangers naturels dans l'aménagement du territoire du canton suisse des Grisons}

Dans le cadre de la couverture des besoins existentiels, l'aménagement du territoire est appelé à participer de façon permanente à la protection des hommes et de leurs biens contre les dangers naturels. Le but d'une prévention passive effective contre les dangers naturels consiste à réduire le risque de danger pour l'homme et ses biens matériels jusqu'à un risque relictuel, en affinant proportionnellement les exigences économiques, sociales et écologiques par des mesures d'aménagement appropriées, en vue de promouvoir un espace économique et de vie satisfaisant. A partir de l'exemple de la Suisse et de son canton des Grisons, la présente contribution montre que l'aménagement du territoire est parfaitement à même d'assumer une telle mission de prévoyance.

Dipl.-Geogr. Kerstin Sauerbrey, Lehrstuhl für Wirtschafts- und Sozialgeographie der Friedrich-Alexander-Universität Erlangen-Nürnberg, Lange Gasse 20, D-90403 Nürnberg.

e-mail: kerstin.sauerbrey@wiso.uni-erlangen.de

Manuskripteingang/received/manuscrit entré le 5.8.2004

Annahme zum Druck/accepted for publication/accepté pour l'impression: 8.3.2005 